\title{
CORRECTION TO A THEOREM OF MINE
}

\author{
R. N. SEN
}

Abstract. One of the theorems published by me in a previous paper turned out to be incorrect. That theorem is replaced in this note by a corrected one.

In 1966 I published the following theorem [4, Theorem 3, p. 882]: (In what follows $C_{n}$ denotes an $n$-dimensional conformally-flat Riemannian space and $C_{n}^{\prime}$ a $C_{n}$ of class one.)

"The coordinates of any $C_{n}^{\prime}$ may be so chosen that its metric assumes the normal form

$$
d s^{2}=\sum_{i}\left(d x^{i}\right)^{2} /[f(\theta)]^{2}, \quad \theta=\sum_{i}\left(x^{i}\right)^{2},
$$

where $f$ is any real analytic function of $\theta$ subject to the restriction

$$
(n-1) f f^{\prime}+\theta f f^{\prime \prime}-(n-1) \theta f^{\prime 2} \neq 0, \quad\left(f^{\prime}=d f / d \theta, \text { etc. }\right) "
$$

In a recent paper [1] G. M. Lancaster has proved that this theorem is incorrect by showing that the above metric does not cover a certain type of $C_{n}^{\prime}$. The purpose of this note is to point out that the metric covers a type of $C_{n}^{\prime}$ although it does not cover all $C_{n}^{\prime}$, and also to give a correct form of the theorem. Before doing so $I$ have to say that on checking an error in a previous paper of mine [2, equations (1.8), p. 107], the referee of the present note has drawn my attention to the fact that the restriction in the above theorem applies when $f^{\prime \prime} \neq 0$; and at the same time he has given a straightforward solution of

$$
(n-1) f f^{\prime}+\theta f f^{\prime \prime}-(n-1) \theta f^{\prime 2}=0, \text { where } f^{\prime \prime} \neq 0,
$$

as

$$
f(\theta)=\theta /\left[K_{1}+(n-2) K_{2} \theta^{n-2}\right]^{1 /(n-2)}, \quad K_{1}>0 .
$$

I heartily thank the referee for the pains and the interest he has taken in the paper. A correct form of the theorem which must replace the above theorem may then be stated as follows $\left(f^{\prime}=d f / d \theta\right.$, etc.):

$A C_{n}(n>3)$ with metric

Received by the editors August 6, 1969 and, in revised form, January 15, 1970.

AMS 1970 subject classifications. Primary 53B20.

Key words and phrases. Conformally-flat Riemannian space, Gauss-Codazzi equations. 


$$
d s^{2}=\sum_{i}\left(d x^{i}\right)^{2} /[f(\theta)]^{2}, \quad \theta=\sum_{i}\left(x^{i}\right)^{2},
$$

where $f(\theta)$ is any real analytic function of $\theta$ subject to the restriction that when $f^{\prime \prime} \neq 0$,

$f(\theta) \neq \theta /\left[K_{1}+(n-2) K_{2} \theta^{n-2}\right]^{1 /(n-2)}, \quad K_{1}>0, \quad K_{2}$ being constants, is a $C_{n}^{\prime}$. The metric covers the case of space of constant curvature when $f^{\prime \prime}=0$.

That the $C_{n}$ is a $C_{n}^{\prime}$ is proved straightway by showing that it satisfies the Gauss-Codazzi equations. In fact, referring to my paper $[4$, p. 882$]$ mentioned at the outset, it is not difficult to see that the $C_{n}$ satisfies equations (7), namely

$$
R_{h i j k}=\frac{R_{h j} R_{i k}-R_{h k} R_{i j}}{(n-2)\left\{(n-2) \rho^{2}+\rho \bar{\rho}\right\}}-\frac{\rho \bar{\rho}}{n-2}\left(g_{h j} g_{i k}-g_{h k} g_{i k}\right),
$$

where $\rho^{2}$ and $\rho \bar{\rho}$ are given by equations (10), namely

$$
\rho^{2}=4 f^{\prime}\left(f-\theta f^{\prime}\right), \quad \rho \bar{\rho}=4\left(f f^{\prime}+\theta f f^{\prime \prime}-\theta f^{\prime 2}\right) .
$$

We have only to take now the second fundamental tensor $b_{i j}$ as

$$
b_{i j}=-\frac{1}{n-2}\left(R_{i j} / \rho+\bar{\rho} g_{i j}\right)
$$

and establish the Gauss-Codazzi equations in exactly the same way as they have been done in a previous paper of mine $[3$, equations (3.8), (5.1)].

\section{REFERENCES}

1. G. M. Lancaster, Note on a theorem by Sen, Proc. Amer. Math. Soc. 19 (1968), 1119-1122. MR 38 \#634.

2. R. N. Sen, On a type of Riemannian space conformal to a flat space, J. Indian Math. Soc. 21 (1957), 105-114. MR 20 \#868.

3. - Conformally euclidean space of class one, Indian J. Math. 6 (1964), 93103. MR 31 \#3977.

4. - On conformally-flat Riemannian space of class one, Proc. Amer. Math. Soc. 17 (1966), 880-883. MR 34 \#753.

Calcutta University, Calcutta, India 